\title{
Stability of a tilt-controlling axial gap self-bearing motor with single-stator
}

\author{
Satoshi UENO*, Ryo ISEKI* and Changan JIANG* \\ * Department of Mechanical Engineering, College of Science and Engineering, Ritsumeikan University \\ 1-1-1 Noji-higashi, Kusatsu, Shiga 525-8577, Japan \\ E-mail: sueno@se.ritsumei.ac.jp
}

Received: 31 May 2016; Revised: 21 February 2017; Accepted: 20 June 2017

\begin{abstract}
This paper discusses the stability of a tilt-controlling axial gap self-bearing motor with a single stator. The motor consists of a stator and a disc rotor and is capable of controlling motor torque, axial position, and tilt angles. The stator has six coils, which are driven by separate amplifiers. By adding two types of current (a motor current, which has the same poles as the rotor, and a tilt control current, which has plus two or minus two poles of the rotor), four degrees of freedom of the rotor can be actively controlled. In this paper, the motor torque, axial force, tilt torques, and radial forces are theoretically analyzed and their control methods of them are derived. The stability of the rotor is discussed and the influence of the position of the center of gravity is shown. The experimental results confirm the analytical results, and it is shown that the stable levitation and rotation are achieved with 2-pole and 4-pole rotors.
\end{abstract}

Key words : Axial gap self-bearing motor, Magnetic bearing, Tilt control, Stability, Rotation test

\section{Introduction}

Magnetic bearing motors are advantageous because they do not exhibit frictional loss and require no lubrication; however, they are not widely used owing to their high cost and large size. To solve these problems, an axial gap selfbearing motor (ASBM) has been proposed (Ueno and Okada, 2000, and Nguyen and Ueno, 2011). The ASBM produces rotation torque and controls axial position simultaneously using single or double stator structure. It can remove a thrust active magnetic bearing, then the cost and size can be reduced. A rotary pump using ASBM has been developed (Okamura, et al., 2010 and Matsuoka, et al., 2014), but it cannot support other axes; hence, additional magnetic bearings are required, such as permanent magnet repulsive-type bearings and superconducting magnetic bearings.

One-axis actively controlled self-bearing motors have been proposed by many research groups. Asama and Chiba (2014) developed a disk type bearingless motor using single coreless stator. Bauer and Amrhein (2012) and Sugimoto, et al. (2016) proposed cylindrical stator and rotor structures for controlling axial force and motor torque. The radial motions of these motors are supported by permanent magnet repulsive-type magnetic bearings.

The requirement of radial magnetic bearings leads to a complex structure and requires special design efforts. Moreover, it is difficult to rotate above the critical speed due to a lack of damping force. Mitterhofer and Jungmayr (2016) improved passive stability for a bearingless disk motor using additional tilt stabilization coil. However, this method is not effective in low rotating speed. To solve these problems, it is necessary to increase the active control axes. An increasing number of the active controlled axis requires higher cost. However, if a compact size and large damping ratio are more significant than the cost rise, the active control can be regarded as a good solution. By adding the motor current and tilt control current using plus two or minus two rotor poles in a similar to radial bearingless motor (Chiba, et al., 2005), tilt torques are generated. A five-axis actively controlling disc motor has been proposed by Osa, et al. (2013) and Kurita, et al. (2016). A three-axis actively controlling self-bearing motor has been proposed by Sumino and Ueno (2014a) These studies have reported on double stator-type permanent magnet motors, which have two stators at both sides of the rotor. Tomita and et al. (1997) also reported a three-axis active controlling disc motor, which consists of a permanent magnet 
motor and reluctance motor. In this motor tilt motions were controlled by the reluctance motor. A single stator-type motor has been reported by Sumino and Ueno (2014b). In that report, successful levitation and rotation were only confirmed with a 4-pole rotor, the stability problem in a 2-pole rotor was pointed out. Moreover, a theoretical analysis was carried out only on axial force, motor torque, and tilt torques, whereas radial forces were not derived.

In this paper, radial forces are analyzed theoretically by using a simple current sheet model. The Lorentz force is considered to derive the radial forces. The control methods of the axial force, motor torque, and tilt torques are introduced for 2-pole and 4-pole rotors. Then, equations of motions are derived, and the stability of the rotor is discussed. The results show that the stability is affected by the position of the center of gravity of the rotor. The results are confirmed by experiments using rotors with different shapes, and it is shown that stable levitation and rotation are achieved with 2-pole and 4-pole rotors.

\section{Tilt-Controlling Axial Gap Self-Bearing Motor}

A schematic drawing of a tilt-controlling ASBM is shown in Fig. 1. The ASBM consists of a stator, a rotor, and sensors. The ASBM generates a rotating torque and controls the axial position and tilt angles of the rotor. The radial directions are supported by the passive stiffness owing to the attractive force between the stator and the rotor. Displacement and angle sensors are used to measure the axial displacement, tilt angles, and angular position of the rotor. In this paper, the stator of the ASBM consists of six concentrated windings and an iron core. Each of the coils is excited by separate power amplifiers. The rotor consists of permanent magnets and an iron core. 2-pole and 4-pole rotors are considered in this paper.

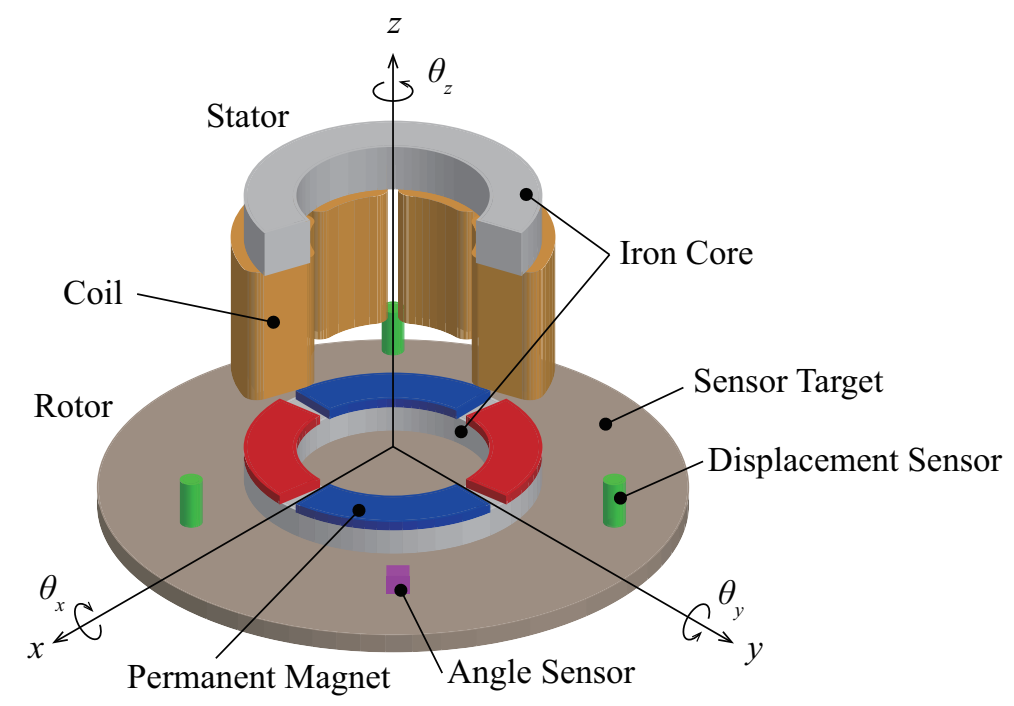

Fig. 1 Schematic drawing of a tilt-controlling ASBM. A stator is set on the upper side of a rotor and controls motor torque, axial position and tilt angles. The radial position of the rotor is passively supported by the attractive force between the stator and the rotor.

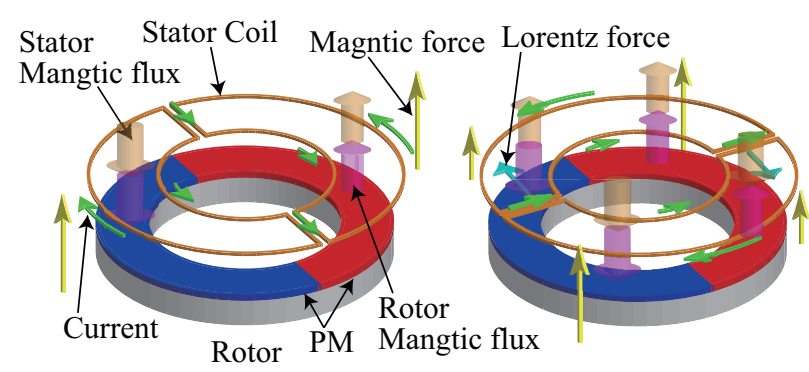

(a) 2-pole $a$-winding

(b) 2-pole $b$-winding

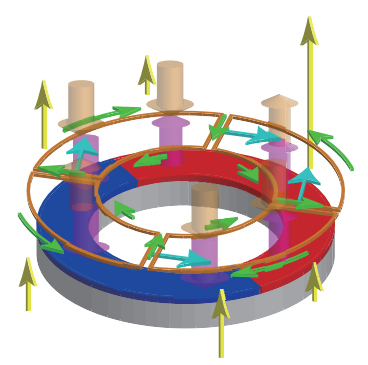

(c) 4-pole $a$-winding

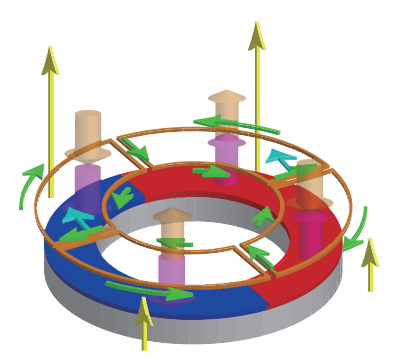

(d) 4-pole $b$-winding

Fig. 2 Generation of motor torque, axial force, and tilt torques for a 2-pole rotor. 2-pole current generates both motor torque and axial force. 4-pole current generates not only tilt torques but also radial forces. 


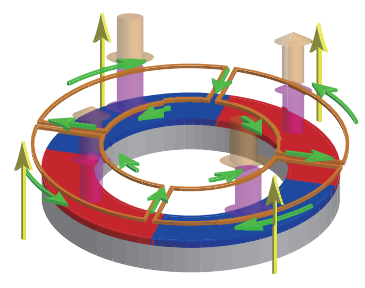

(a) 4-pole $a$-winding

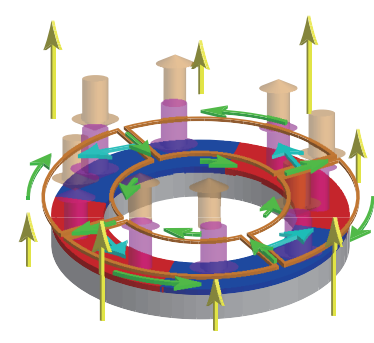

(b) 4-pole $b$-winding

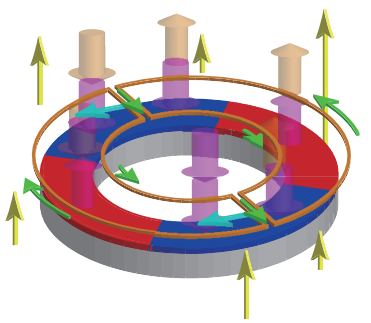

(c) 2-pole $a$-winding

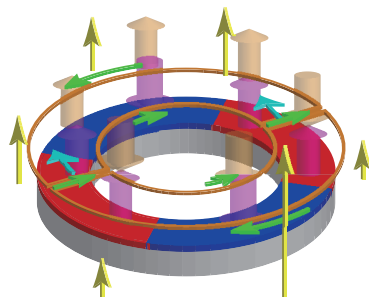

(d) 2-pole $b$-winding

Fig. 3 Generation of motor torque, axial force, and tilt torques for a 4-pole rotor. 4-pole current generates both motor torque and axial force. 2-pole current generates not only tilt torques but also radial forces. The direction of the radial force in (c) and the tilt moment in (d) are the opposite of those in Fig. 2 (c) and (d), respectively.

Figure 2 shows the generation of motor torque and suspension forces for the 2-pole rotor. For simplicity, the stator core is omitted and the stator coils are transformed to equivalent windings, as follows: 2-pole $a$-winding, which produces the same directional 2-pole magnetic flux as the rotor permanent magnet; 2-pole $b$-winding, which has a 90 degrees phase difference from the 2-pole $a$-winding; 4-pole $a$-winding, which produces 4-pole magnetic flux and the N-pole is in the same angular position as the N-pole of the permanent magnet; and 4-pole $b$-winding, which has a 45 degrees phase difference from 4-pole $a$-axis. The equivalent windings are shown by ochre lines. The direction of currents is indicated by green arrows while the magnetic fluxes produced by the permanent magnet and the coil currents are shown by translucent magenta and ochre arrows, respectively. The magnetic attractive forces and Lorentz forces produced by the permanent magnets and coil currents are shown by yellow and cyan arrows, respectively.

The axial force is controlled by the 2-pole $a$-winding current as shown in Fig. 2 (a) and the motor torque is controlled by the 2-pole $b$-winding current as shown in Fig. 2 (b). The 2-pole $b$-winding current produces not only the motor torque but also the axial force. The undesirable axial force can be controlled by the 2-pole $a$-winding current. The tilt torques are controlled by the magnetic force difference between opposite poles as shown in Fig. 2 (c) and (d). The 4-pole currents generate not only the tilt moments but also radial forces by Lorentz force.

Figure 3 shows the generation of the motor torque and suspension forces for the 4-pole rotor. The principles are the same as for the 2-pole rotor. In this case, 4-pole currents control the axial force and motor torque, and 2-pole currents control tilt torques. It should be noted that the direction of radial force and tilt moment are not the same as in the 2-pole rotor.

\section{Analysis of Motor Torque, Axial Force, Tilt Torques and Radial Forces}

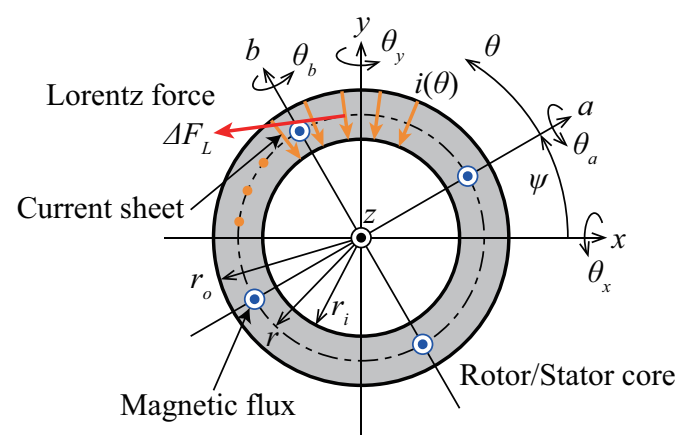

(a) Top View
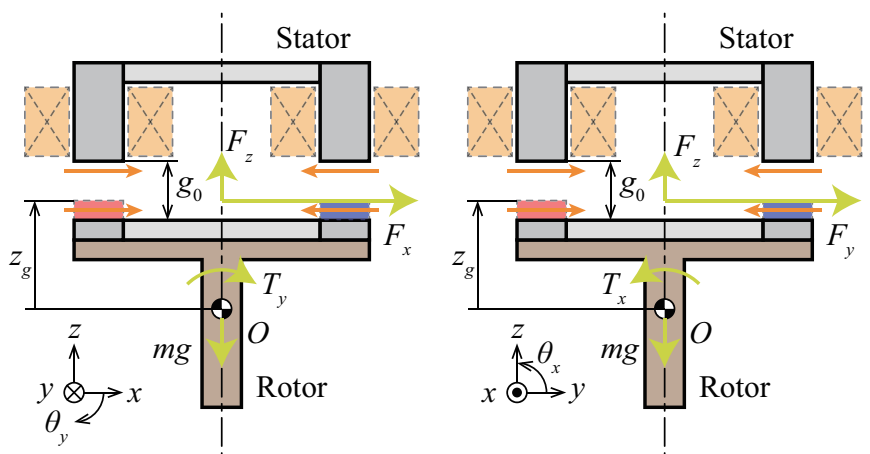

(b) Side View

Fig. 4 Rotational Coordinates $a-b-z$ are used for the analysis of the motor torque, axial and radial forces and tilt torques. An origin is set at the center of the gravity of the rotor. Stator coils and rotor magnets are replaced by equivalent current sheets.

For the simplicity of analysis, non-salient rotor and stator as well as current sheet windings are assumed, and the rotor displacement is not considered. Moreover, the magnetic resistance in the core and leakage flux is neglected. The rotor permanent magnet is replaced by the current sheet with equivalent current. Although the actual motor has different 
stator structure, we adopt the simplified model to find out an essential factor of the stability. The coordinate system is shown in Fig. 4. The angle of rotation of the rotor is defined as $\psi$, and the coordinate system on the rotor is defined as the $a-b$ axis. The equivalent current distributions of the rotor magnet are assumed to be sinusoidal, and they are expressed as

$$
i_{P r}(\theta)=\frac{P}{2} I_{P r} \sin \left(\frac{P}{2} \theta\right)
$$

where $P=2$ or 4 is pole number and $I$ denotes an amplitude. The stator current distributions are assumed as

$$
\begin{aligned}
& i_{P a}(\theta)=\frac{P}{2} I_{P a} \sin \left(\frac{P}{2} \theta\right) \\
& i_{P b}(\theta)=-\frac{P}{2} I_{P b} \cos \left(\frac{P}{2} \theta\right)
\end{aligned}
$$

Magnetic flux density distributions in the air gap can be expressed as

$$
\begin{aligned}
& B_{P r}(\theta)=\frac{4 \mu_{0} N}{g_{0}} \cos \left(\frac{P}{2} \theta\right) I_{P r} \\
& B_{P a}(\theta)=\frac{4 \mu_{0} N}{g_{0}} \cos \left(\frac{P}{2} \theta\right) I_{P a} \\
& B_{P b}(\theta)=\frac{4 \mu_{0} N}{g_{0}} \sin \left(\frac{P}{2} \theta\right) I_{P b}
\end{aligned}
$$

where $g_{0}$ is the air gap between the stator and the rotor core, $\mu_{0}$ is the permeability of air, and $N$ is equivalent turn number of windings. Then, the resultant magnetic flux distribution is calculated as

$$
B(\theta)=B_{2 r}(\theta)+B_{2 a}(\theta)+B_{2 b}(\theta)+B_{4 r}(\theta)+B_{4 a}(\theta)+B_{4 b}(\theta)
$$

The magnetic force of small area $\Delta S$ is calculated by

$$
\Delta F_{M}=\frac{B(\theta)^{2}}{2 \mu_{0}} \Delta S=\frac{B(\theta)^{2}}{2 \mu_{0}} \frac{S}{2 \pi} d \theta
$$

where $S=\pi\left(r_{o}^{2}-r_{i}^{2}\right)$, and $r_{o}$ and $r_{i}$ are outer and inner radii of the rotor, respectively. Then, the axial force becomes

$$
F_{z}=\int_{0}^{2 \pi} \Delta F_{M}=\frac{4 \mu_{0} S N^{2}}{g_{0}^{2}}\left\{\left(I_{2 r}+I_{2 a}\right)^{2}+I_{2 b}^{2}+\left(I_{4 r}+I_{4 a}\right)^{2}+I_{4 b}^{2}\right\}
$$

Tilt torques are calculated as

$$
\begin{aligned}
& T_{a}=\int_{0}^{2 \pi} r \sin (\theta) \Delta F_{M}=\frac{4 \mu_{0} r S N^{2}}{g_{0}^{2}}\left(I_{2 r} I_{4 b}-I_{4 r} I_{2 b}+I_{2 a} I_{4 b}-I_{4 a} I_{2 b}\right) \\
& T_{b}=-\int_{0}^{2 \pi} r \cos (\theta) \Delta F_{M}=\frac{4 \mu_{0} r S N^{2}}{g_{0}^{2}}\left(-I_{2 r} I_{4 a}-I_{4 r} I_{2 a}-I_{2 a} I_{4 a}-I_{2 b} I_{4 b}-I_{4 r} I_{2 r}\right)
\end{aligned}
$$

where $r$ is mean radius of the rotor.

Lorentz force of small winding is calculated by

$$
\Delta F_{L}=B(\theta)\left(r_{o}-r_{i}\right) N\left\{i_{2 r}(\theta)+i_{4 r}(\theta)-i_{2 a}(\theta)-i_{2 b}(\theta)-i_{4 a}(\theta)-i_{4 b}(\theta)\right\} d \theta
$$

In the actual case, the magnetic flux density depends on the distance from the surface of the permanent magnets, hence note that this calculation can be applied to only ideal case. Motor torque becomes

$$
T_{z}=\int_{0}^{2 \pi} r \Delta F_{L}=\frac{4 \mu_{0} S N^{2}}{g_{0}}\left(I_{2 r} I_{2 b}+2 I_{4 r} I_{4 b}\right)
$$

Radial forces become

$$
\begin{aligned}
& F_{a}=-\int_{0}^{2 \pi} \Delta F_{L} \sin (\theta)=\frac{3 \mu_{0} S N^{2}}{g_{0} r}\left(I_{2 r} I_{4 a}-I_{4 r} I_{2 a}-\frac{I_{2 r} I_{4 r}}{3}+\frac{I_{2 a} I_{4 a}}{3}+\frac{I_{2 b} I_{4 b}}{3}\right) \\
& F_{b}=\int_{0}^{2 \pi} \Delta F_{L} \cos (\theta)=\frac{3 \mu_{0} S N^{2}}{g_{0} r}\left(I_{2 r} I_{4 b}+I_{4 r} I_{2 b}+\frac{I_{2 a} I_{4 b}}{3}-\frac{I_{4 a} I_{2 b}}{3}\right)
\end{aligned}
$$

In this self-bearing motor, although the axial position, motor torque and tilt motions are actively controlled, the radial motions are not. The radial motions are stabilized by using passive stability due to the attractive force between the rotor 
and stator. Defining axial force control current as $i_{z}$, motor current as $i_{m}$, and tilt control currents as $i_{t x}$ and $i_{t y}$, the stator current for the 2-pole rotor (in this case, the 4-pole rotor current $I_{4 r}=0$ ) is obtained as

$$
\left[\begin{array}{c}
I_{2 a} \\
I_{2 b} \\
I_{4 a} \\
I_{4 b}
\end{array}\right]=\left[\begin{array}{cccc}
1 & 0 & 0 & 0 \\
0 & 1 & 0 & 0 \\
0 & 0 & 0 & -1 \\
0 & 0 & 1 & 0
\end{array}\right]\left[\begin{array}{c}
i_{z} \\
i_{m} \\
i_{t a} \\
i_{t b}
\end{array}\right]=\left[\begin{array}{cccc}
1 & 0 & 0 & 0 \\
0 & 1 & 0 & 0 \\
0 & 0 & \sin \psi & -\cos \psi \\
0 & 0 & \cos \psi & \sin \psi
\end{array}\right]\left[\begin{array}{c}
i_{z} \\
i_{m} \\
i_{t x} \\
i_{t y}
\end{array}\right]
$$

where $i_{t a}$ and $i_{t b}$ are the tilt control current around $a$ - and $b$-axis, and are expressed as

$$
\left[\begin{array}{c}
i_{t a} \\
i_{t b}
\end{array}\right]=\left[\begin{array}{cc}
\cos \psi & \sin \psi \\
-\sin \psi & \cos \psi
\end{array}\right]\left[\begin{array}{c}
i_{t x} \\
i_{t y}
\end{array}\right]
$$

Then, the forces and torques can be approximated around $i_{z 0}$ and $i_{t x}=i_{t y}=i_{m}=0$ as

$$
\begin{aligned}
& F_{z} \approx \frac{4 \mu_{0} S N^{2}}{g_{0}^{2}}\left(I_{2 r}+i_{z 0}\right)^{2}+\frac{8 \mu_{0} S N^{2}}{g_{0}^{2}}\left(I_{2 r}+i_{z 0}\right)\left(i_{z}-i_{z 0}\right) \equiv F_{z 0}+K_{z}\left(i_{z}-i_{z 0}\right) \\
& T_{z}=\frac{4 \mu_{0} S N^{2} I_{2 r}}{g_{0}} i_{m} \equiv K_{m} i_{m} \\
& F_{x} \approx-\frac{3 \mu_{0} S N^{2}}{g_{0} r}\left(I_{2 r}+\frac{i_{z 0}}{3}\right) i_{t y} \equiv-K_{r} i_{t y} \\
& F_{y} \approx \frac{3 \mu_{0} S N^{2}}{g_{0} r}\left(I_{2 r}+\frac{i_{z 0}}{3}\right) i_{t x} \equiv K_{r} i_{t x} \\
& T_{x} \approx \frac{4 \mu_{0} r S N^{2}}{g_{0}^{2}}\left(I_{2 r}+i_{z 0}\right) i_{t x} \equiv K_{t} i_{t x} \\
& T_{y} \approx \frac{4 \mu_{0} r S N^{2}}{g_{0}^{2}}\left(I_{2 r}+i_{z 0}\right) i_{t y} \equiv K_{t} i_{t y}
\end{aligned}
$$

For the 4-pole rotor $\left(I_{2 r}=0\right)$,

$$
\left[\begin{array}{c}
I_{2 a} \\
I_{2 b} \\
I_{4 a} \\
I_{4 b}
\end{array}\right]=\left[\begin{array}{cccc}
0 & 0 & 0 & -1 \\
0 & 0 & -1 & 0 \\
1 & 0 & 0 & 0 \\
0 & 1 & 0 & 0
\end{array}\right]\left[\begin{array}{c}
i_{z} \\
i_{m} \\
i_{t a} \\
i_{t b}
\end{array}\right]=\left[\begin{array}{cccc}
0 & 0 & \sin \psi & -\cos \psi \\
0 & 0 & -\cos \psi & -\sin \psi \\
1 & 0 & 0 & 0 \\
0 & 1 & 0 & 0
\end{array}\right]\left[\begin{array}{c}
i_{z} \\
i_{m} \\
i_{t x} \\
i_{t y}
\end{array}\right]
$$

The forces and torques are

$$
\begin{aligned}
& F_{z} \approx \frac{4 \mu_{0} S N^{2}}{g_{0}^{2}}\left(I_{4 r}+i_{z 0}\right)^{2}+\frac{8 \mu_{0} S N^{2}}{g_{0}^{2}}\left(I_{4 r}+i_{z 0}\right)\left(i_{z}-i_{z 0}\right) \equiv F_{z 0}+K_{z}\left(i_{z}-i_{z 0}\right) \\
& T_{z}=\frac{8 \mu_{0} S N^{2} I_{4 r}}{g_{0}} i_{m} \equiv K_{m} i_{m} \\
& F_{x} \approx \frac{3 \mu_{0} S N^{2}}{g_{0} r}\left(I_{4 r}-\frac{i_{z 0}}{3}\right) i_{t y} \equiv K_{r} i_{t y} \\
& F_{y} \approx-\frac{3 \mu_{0} S N^{2}}{g_{0} r}\left(I_{4 r}-\frac{i_{z 0}}{3}\right) i_{t x} \equiv-K_{r} i_{t x} \\
& T_{x} \approx \frac{4 \mu_{0} r S N^{2}}{g_{0}^{2}}\left(I_{4 r}+i_{z 0}\right) i_{t x} \equiv K_{t} i_{t x} \\
& T_{y} \approx \frac{4 \mu_{0} r S N^{2}}{g_{0}^{2}}\left(I_{4 r}+i_{z 0}\right) i_{t y} \equiv K_{t} i_{t y}
\end{aligned}
$$

These results show that there are coupling terms between the radial forces and tilt torques, and the radial force gains of the 4-pole rotor have opposite signs to the 2-pole rotor. The influence of the coupling terms on the stability is investigated. The distance from the center of gravity (COG) of the rotor to the surface of the rotor permanent magnet, which is the acting point of radial force, is defined as $z_{g}$. When the COG is above the permanent magnet, $z_{g}$ takes negative value. For simplicity, assuming constant axial force and zero motor current for simplicity, the equations of the radial 
motions of the 2-pole rotor are expressed as

$$
\begin{aligned}
m \ddot{x}=F_{x}-K_{r p}\left(x+z_{g} \theta_{y}\right) & =-K_{r p} x-\left(K_{r p} z_{g} \theta_{y}+K_{r} i_{t y}\right) \\
I_{r} \ddot{\theta}_{y}=T_{y}+K_{t n} \theta_{y}+z_{g}\left\{F_{x}-K_{r p}\left(x+z_{g} \theta_{y}\right)\right\} & =-K_{r p} z_{g} x+\left(K_{t n}-K_{r p} z_{g}^{2}\right) \theta_{y}+\left(K_{t}-K_{r} z_{g}\right) i_{t y} \\
m \ddot{y}=F_{y}-K_{r p}\left(y-z_{g} \theta_{x}\right) & =-K_{r p} y+\left(K_{r p} z_{g} \theta_{x}+K_{r} i_{t x}\right) \\
I_{r} \ddot{\theta}_{x}=T_{x}+K_{t n} \theta_{x}-z_{g}\left\{F_{y}-K_{r p}\left(y-z_{g} \theta_{x}\right)\right\} & =K_{r p} z_{g} y+\left(K_{t n}-K_{r p} z_{g}^{2}\right) \theta_{x}+\left(K_{t}-K_{r} z_{g}\right) i_{t x}
\end{aligned}
$$

where $m$ is the mass of the rotor, $I_{r}$ is the moment of inertia of the rotor around radial axis, $K_{t n}$ is negative stiffness for tilt motion, and $K_{r p}$ is passive stiffness for the radial motion due to the attractive force between the rotor and stator. For the 4-pole rotor, the equations of motions are

$$
\begin{aligned}
m \ddot{x}=F_{x}-K_{r p}\left(x+z_{g} \theta_{y}\right) & =-K_{r p} x-\left(K_{r p} z_{g} \theta_{y}-K_{r} i_{t y}\right) \\
I_{r} \ddot{\theta}_{y}=T_{y}+K_{t n} \theta_{y}+z_{g}\left\{F_{x}-K_{r p}\left(x+z_{g} \theta_{y}\right)\right\} & =-K_{r p} z_{g} x+\left(K_{t n}-K_{r p} z_{g}^{2}\right) \theta_{y}+\left(K_{t}+K_{r} z_{g}\right) i_{t y} \\
m \ddot{y}=F_{y}-K_{r p}\left(y-z_{g} \theta_{x}\right) & =-K_{r p} y+\left(K_{r p} z_{g} \theta_{x}-K_{r} i_{t x}\right) \\
I_{r} \ddot{\theta}_{x}=T_{x}+K_{t n} \theta_{x}-z_{g}\left\{F_{y}-K_{r p}\left(y-z_{g} \theta_{x}\right)\right\} & =K_{r p} z_{g} y+\left(K_{t n}-K_{r p} z_{g}^{2}\right) \theta_{x}+\left(K_{t}+K_{r} z_{g}\right) i_{t x}
\end{aligned}
$$

According to these results, the stability of the tilt motions are affected by the position of the COG $z_{g}$ and radial force gain $K_{r}$. In the 2-pole rotor, a lower COG decreases the gain of the tilt torques with respect to the tilt control current, while in the 4-pole rotor, it is the opposite. This effect becomes more significant as the radial force gain becomes larger, and hence, the 2-pole rotor is significantly more affected than the 4-pole rotor from Eqs. (20), (21), (27), and (28). Moreover, since there are coupling terms between radial and tilt motions, then the COG position affects them.

\section{Experimental Setup and Control System}
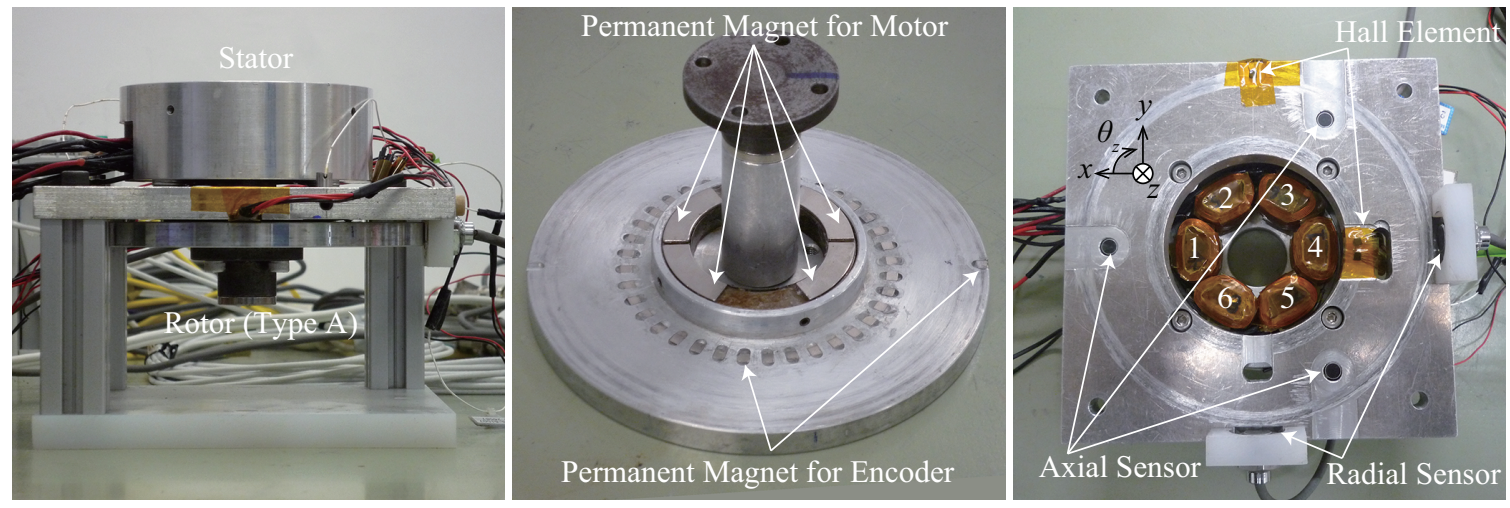

Fig. 5 Photographs of the experimental device. Left: test motor with Type A rotor. Center: rotor (Type B). Right: stator and sensors.

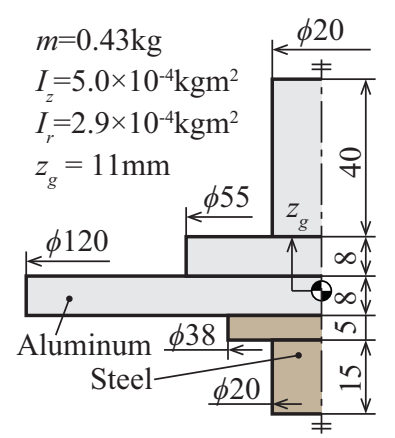

Type A

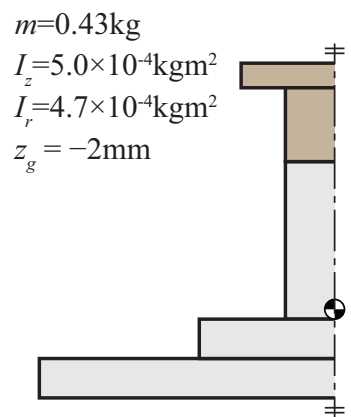

Type B

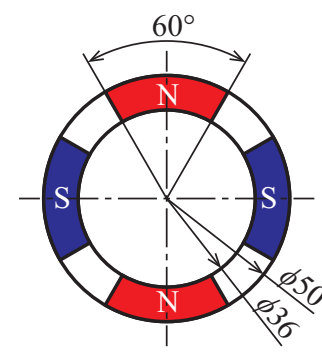

4-pole Rotor

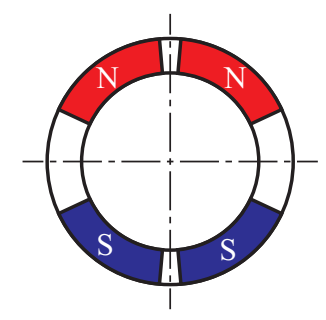

2-pole Rotor

Fig. 6 Dimensions and specifications of the rotor. Two shapes were tested. The two drawings on the right show the arrangement of permanent magnets of the 4-pole and 2-pole rotors.

Figure 5 shows the photographs of the experimental setup: the entire device, on the left side; the rotor, at the center; the stator and sensors, on the right side. The rotor consists of the motor rotor, sensor target, and permanent magnets for 
encoder. Two types of rotor, which have different centers of gravity as shown in Fig. 6, were tested. The bottom parts of type A were attached on the top of the rotor in type B. The arrangement of the 4- and 2-pole rotors is shown in Fig. 6. The same permanent magnets were used for both rotors. $I_{z}$ is the moment of inertia of the rotor around the rotation axis $z$. The stator consists of an iron core and six concentrated coils. Table 1 shows the parameters of the stator and rotor. The arrangement of coils is shown in Fig. 7. Positive current produces positive magnetic flux, as indicated by the green circles. To create magnetic flux density, as shown in Eqs. (5) and (6), each coil current is expressed as

$$
i_{k}=I_{2 a} \cos \left(\frac{k-1}{3} \pi-\psi\right)+I_{2 b} \sin \left(\frac{k-1}{3} \pi-\psi\right)+I_{4 a} \cos \left(2 \frac{k-1}{3} \pi-2 \psi\right)+I_{4 b} \sin \left(2 \frac{k-1}{3} \pi-2 \psi\right)
$$

where $k=1 \sim 6$ is coil number.

Figure 8 shows the location of the sensors. Three eddy-current type displacement sensors (SENTEC, HA-50S) are installed on the top of the rotor to measure the axial displacement and tilt angle. They are calculated as

$$
\begin{aligned}
z & =g_{0}-\frac{d_{1}+d_{2}+d_{3}}{3}[\mathrm{~m}] \\
\theta_{x} & =\tan ^{-1} \frac{d_{3}-d_{2}}{50 \sqrt{3} \times 10^{-3}}[\mathrm{rad}] \\
\theta_{y} & =\tan ^{-1} \frac{2 d_{1}-d_{2}-d_{3}}{150 \times 10^{-3}}[\mathrm{rad}]
\end{aligned}
$$

where $d_{1}, d_{2}$, and $d_{3}$ are the distances between the sensor top and sensor target, and $g_{0}$ is the gap between the rotor and sensors at floating point. $g_{0}$ was set at $0.5 \mathrm{~mm}$ and the air gap between the stator and rotor was $4.5 \mathrm{~mm}$ at $z=0$. The radial displacements are monitored by two sensors (SENTEC, HA-101S) installed on the side. The radial sensors are not used for control. To measure the angular position of the rotor, three Hall elements (Asahi Kasei Microdvices, HZ-312C) and square-shaped magnets $(\mathrm{NdFeB}, 3 \times 3 \times 1 \mathrm{~mm})$ are attached. The output voltages of the Hall elements are transformed to a pulse wave, and then, the angular position and rotating speed are measured by counting the pulses. A first-order low-pass filter (LPF) is adopted to reduce the quantization error of the rotation speed. The cut-off frequency of the LPF is set to 10 $\mathrm{Hz}$.

Figure 9 shows the control system. A digital signal processor (DSP) is used for control. Analog-digital (A/D) converters read signals from the displacement sensors and Hall elements. The DSP calculates each coil current, and then outputs the current command of each coil via digital-analog (D/A) converters. Power amplifiers, which use a current feedback circuit, supply demand currents for each coil.

The controllers are shown in Fig. 10. Two types of controller are used for motor torque control. Figure 10 (a) shows the open loop torque controller, which does not use the information on the angular position of the rotor. The motor torque is passively produced by the phase difference between the rotor and the stator current. The angular position $\psi$ of the stator current is determined by integrating the reference speed $\omega_{r}$. The axial and tilt positions are controlled by proportionalintegral-derivative (PID) controllers. An approximate derivative is used for the PID controller. The transfer function of the PID controller is

$$
G(s)=k_{p}+\frac{k_{d} s}{\left(1 / \omega_{b}\right) s+1}+\frac{k_{i}}{s}
$$

where $k_{p}$ is proportional gain, $k_{d}$ is differential gain, $\omega_{b}$ is the break angular frequency of the approximate derivative which was set to $2 \pi \times 500[\mathrm{rad} / \mathrm{s}]$, and $k_{i}$ is integral gain. The PID controller is transformed to a discrete transfer function with the sampling time of $0.1 \times 10^{-3}[\mathrm{~s}]$ by a bilinear transformation, and then, it is implemented by the DSP. The control currents $i_{z}, i_{t x}$, and $i_{t y}$ are transformed to the amplitude $I_{2 a}, I_{2 b}, I_{4 a}$, and $I_{4 b}$ by Eq. (16) for the 2-pole rotor or Eq. (24) for the 4-pole rotor. Substituting Eq. (16) into (39), we have currents for the 2-pole rotor as

$$
i_{k}=i_{z} \cos \left(\frac{k-1}{3} \pi-\psi\right)+i_{m} \sin \left(\frac{k-1}{3} \pi-\psi\right)+i_{t x} \sin \left(2 \frac{k-1}{3} \pi-\psi\right)-i_{t y} \cos \left(2 \frac{k-1}{3} \pi-\psi\right)
$$

Table 1 Parameters of the stator and rotor

\begin{tabular}{cc}
\multicolumn{2}{c}{ Rotor and Stator Core } \\
\hline Material & Silicon Steel $(\mathrm{t} 0.1 \mathrm{~mm})$ \\
Rotor & $\phi 50 \times \phi 36 \times 7 \mathrm{~mm}$ \\
Stator & $\phi 50 \times \phi 36 \times 25 \mathrm{~mm}$ \\
Slot & $10 \mathrm{w} . \times 18 \mathrm{~d} . \mathrm{mm}$ \\
\hline
\end{tabular}

\begin{tabular}{cc} 
& Rotor PM \\
\hline Material & NdFeB \\
Magnetization & Perpendicular \\
Shape & Segment \\
Size & $\mathrm{r} 25 \mathrm{~mm} \times \mathrm{r} 18 \mathrm{~mm} \times \mathrm{t} 1 \mathrm{~mm} \times 60^{\circ}$ \\
\hline
\end{tabular}

\begin{tabular}{cc}
\multicolumn{2}{c}{ Stator Coil } \\
\hline Wire & UEW, $\phi 0.5 \mathrm{~mm}$ \\
Turns & 200 \\
Resistance & $1.2 \Omega$ \\
\hline
\end{tabular}




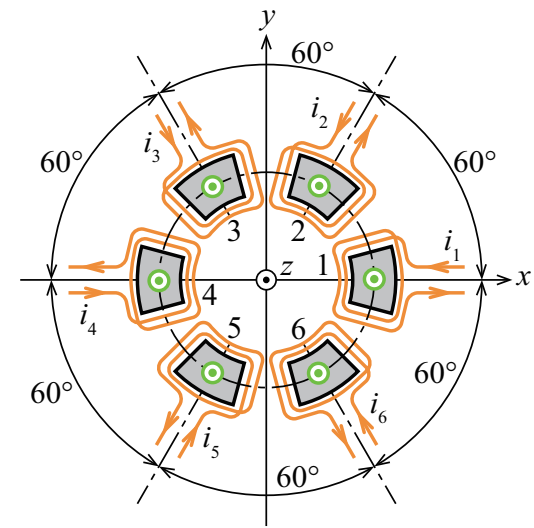

Fig. 7 Coil location. Each coil is driven by the separate amplifiers. Power OP Amps and current feedback circuits were used for the power amplifiers.

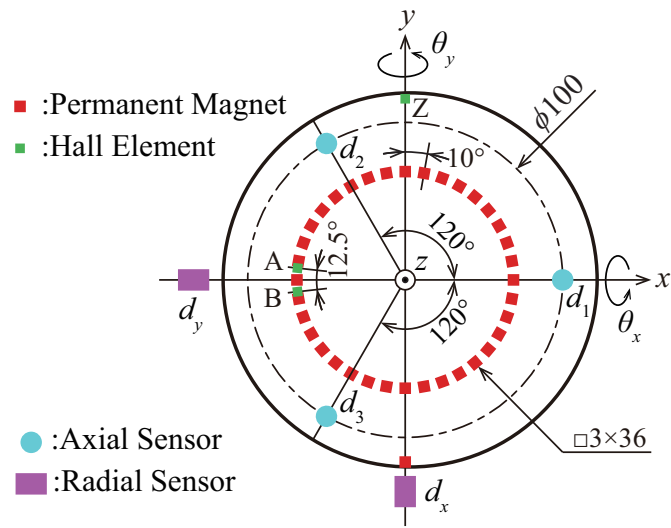

Fig. 8 Sensor location. Five eddy current type displacement sensors and three Hall elements are used for measurement of the rotor's position.

DSP (dSPACE DS1103) Power Amp.

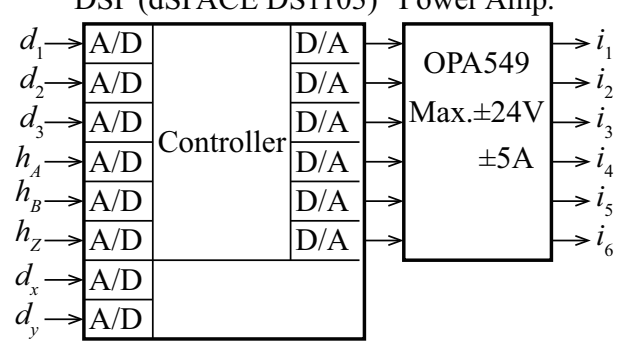

Fig. 9 Control system. DSP is used for the motor and position control.

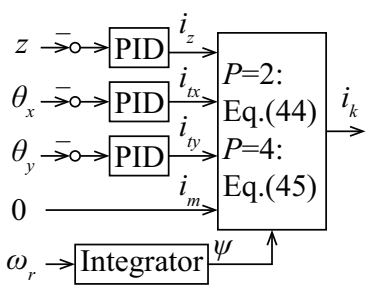

(a) Open Loop Torque Controller

Fig. 10 Controllers. Two types of motor controller are used.

Substituting Eq. (24) into (39), we have currents for the 4-pole rotor as

$$
i_{k}=i_{z} \cos \left(2 \frac{k-1}{3} \pi-2 \psi\right)+i_{m} \sin \left(2 \frac{k-1}{3} \pi-2 \psi\right)-i_{t x} \sin \left(\frac{k-1}{3} \pi-2 \psi\right)-i_{t y} \cos \left(\frac{k-1}{3} \pi-2 \psi\right)
$$

Figure 10 (b) shows the closed loop torque controller. The rotation speed is controlled by a proportional-integral (PI) controller. The measured $\psi$ is used for current calculations.

\section{Experimental Results}

\subsection{Levitation Tests}

First, the results of levitation tests are shown. The gains of the position controllers are listed in Table 2. Small $k_{d}$ values were used to measure the natural frequencies of the tilt motions. Stable levitation was achieved by the 2-pole type $\mathrm{B}$, 4-pole type A, and 4-pole type B, but failed by the 2-pole type A rotor. $i_{z 0}$ of the 2-pole type B, 4-pole type A, and 4-pole type B were $0.71 \mathrm{~A}, 0.95 \mathrm{~A}$, and $0.97 \mathrm{~A}$, respectively. This means that types A and B have almost the same motor characteristics.

The transient responses of the 2-pole type A rotor are shown in Fig. 11. Self-excited vibration was occurred in the $\theta_{x}$ and $y$ axes. Even though the same control gains were used for type A and B rotors, only the type A rotor was unstable. Therefore, one of the reasons for the vibration is weak stability due to the position of COG.

The impulse responses of the 2-pole type B are shown in Fig. 12. These were obtained by injecting pseudo-impulse signals to both $i_{t x}$ and $i_{t y}$. Because of the small differential gains vibrations appeared. When larger differential gains were used, the vibrations were suppressed quickly. The natural frequency and equivalent stiffness of the rotor are shown in Table 3. Natural frequency was calculated by Fast Fourier Transformation of the impulse responses. Equivalent stiffness

Table 2 Parameters of PID controllers for levitation tests. Small $k_{d}$ was used for tilt controllers to measure the natural frequencies of the tilt motions.

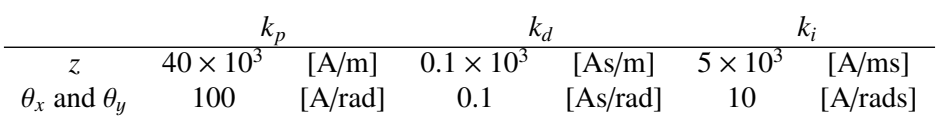




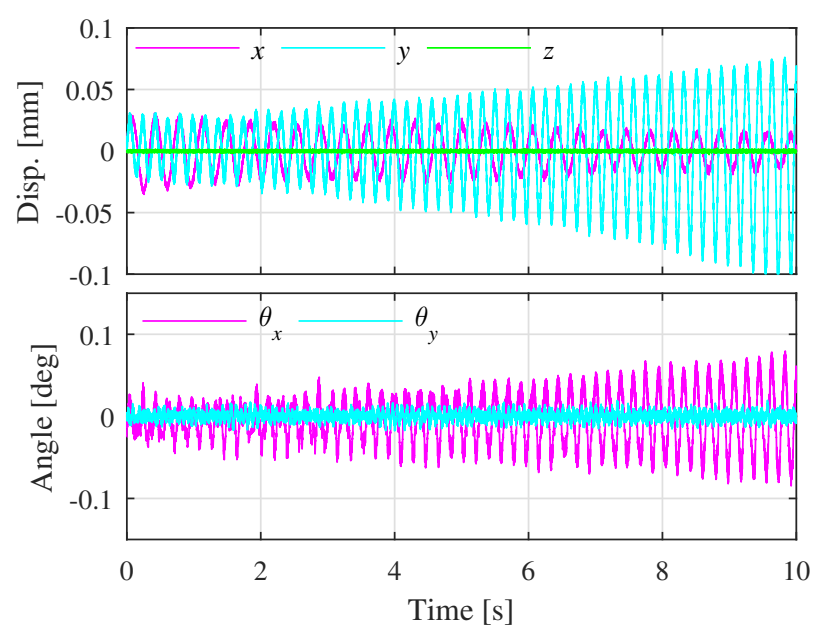

Fig. 11 Levitation test of the 2-pole type A rotor. Self-excited vibration occurred due to the position of COG.

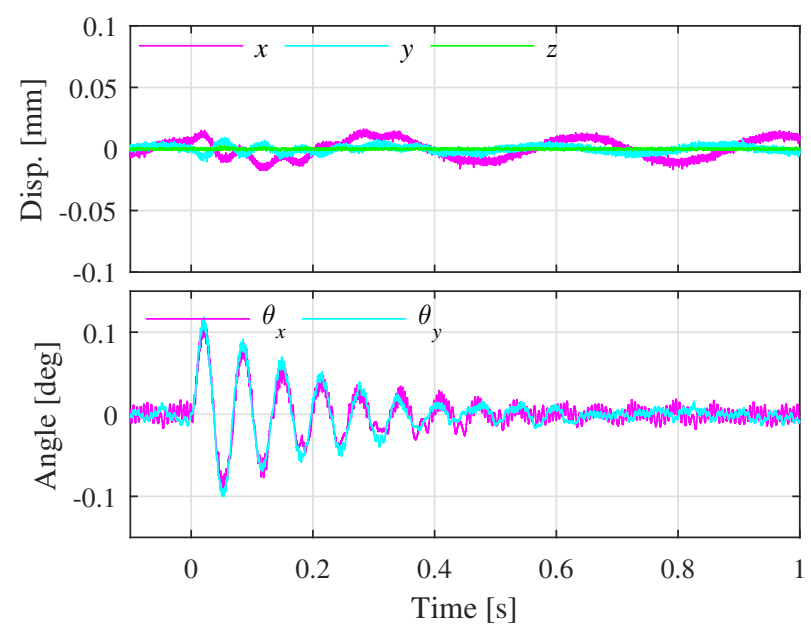

Fig. 13 Impulse response of the 4-pole type A rotor. Stable levitation was achieved.

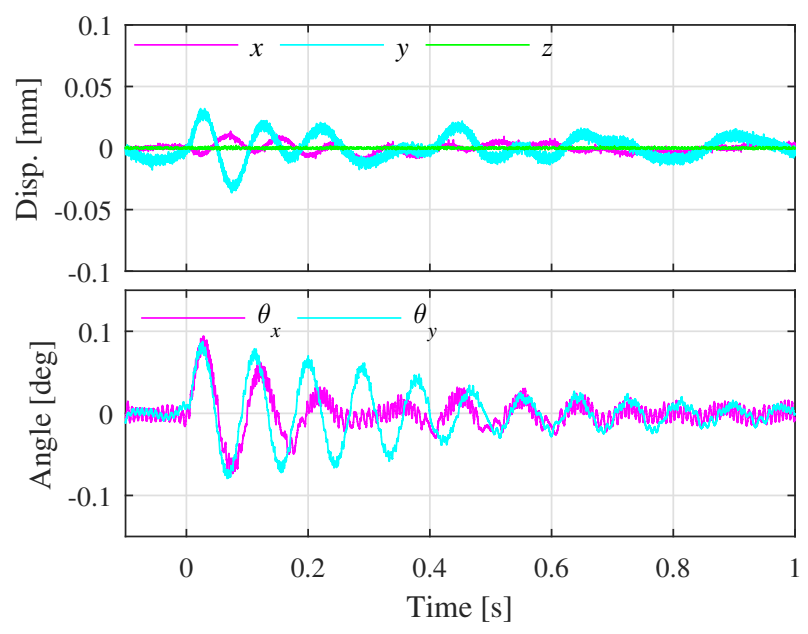

Fig. 12 Impulse response of the 2-pole type B rotor. Stiffness of $\theta_{x}$ is smaller than that of $\theta_{y}$. Radial motions were affected by tilt motions.

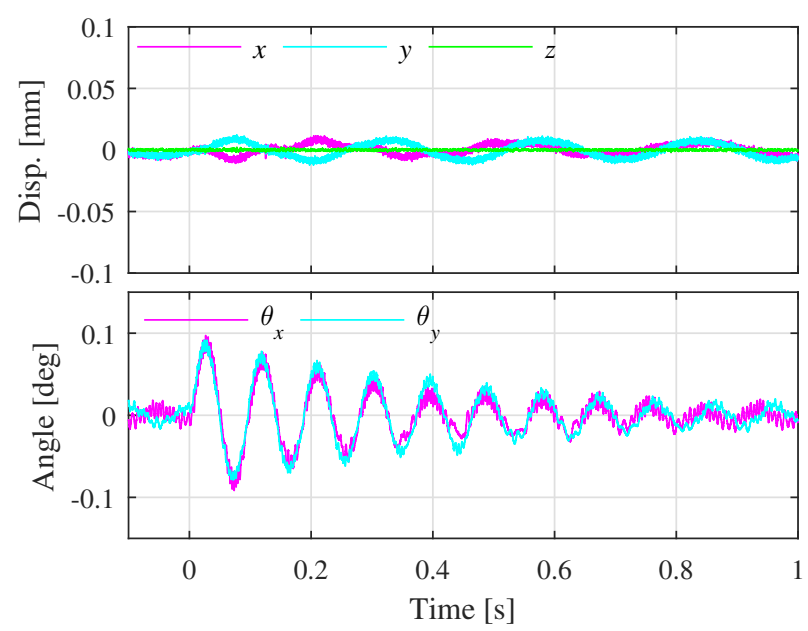

Fig. 14 Impulse response of the 4-pole type B rotor. Stiffness of tilt motions is smaller than those of the type A rotor.

was calculated from the natural frequency and rotor's inertia. In 2-pole rotor, $K_{\theta x}$ and $K_{x}$ is smaller than $K_{\theta y}$ and $K_{y}$, respectively. One of the considered reasons is the unideal magnetic flux density distribution in the air gap, due to the shapes of the permanent magnets and stator slots. Nevertheless, further consideration is needed.

The impulse responses of the 4-pole type A and B rotors are shown in Figs. 13 and 14. The 4-pole rotors could achieve a more stable levitation than the 2-pole rotors, and almost the same characteristics in $\theta_{x}$ and $\theta_{y}$. Natural frequencies and stiffness are shown in Table 3. As expected in Section 3, the type B rotor has smaller stiffness than the type A rotor. The radial stiffness is also different between the 4-pole type A and B. As shown in Eqs. (35) (38), the radial and tilt motions are coupled by $K_{r p} z_{g}$. Therefore, natural frequencies were affected by the COG position, then it is considered that the different radial stiffness was measured.

Next, we estimate the radial force gains $K_{r}$. From Eqs. (34) and (38) and proportional feedback control, the equiva-

Table 3 Natural frequency and equivalent stiffness. They were obtained by the impulse responses excited along each axis. In cases of $\theta_{x}$ and $\theta_{y}$, a disturbance signal was injected to the control current. In the other cases, the rotor was hit by hand.

\begin{tabular}{c|ccccc|ccccc} 
& \multicolumn{6}{|c|}{ Natural Frequency } & \multicolumn{5}{c}{ Equivalent Stiffness } \\
& $\theta_{x}[\mathrm{~Hz}]$ & $\theta_{y}[\mathrm{~Hz}]$ & $x[\mathrm{~Hz}]$ & $y[\mathrm{~Hz}]$ & $\theta_{z}[\mathrm{~Hz}]$ & $K_{\theta x}[\mathrm{Nm} / \mathrm{rad}]$ & $K_{\theta y}[\mathrm{Nm} / \mathrm{rad}]$ & $K_{x}[\mathrm{~N} / \mathrm{m}]$ & $K_{y}[\mathrm{~N} / \mathrm{m}]$ & $K_{\theta z}[\mathrm{Nm} / \mathrm{rad}]$ \\
\hline 2-pole Type B & 9.8 & 11.6 & 2.14 & 4.20 & 1.30 & 1.76 & 2.49 & 77.9 & 300 & 0.0332 \\
4-pole Type A & 15.3 & 14.9 & 2.90 & 3.74 & 1.53 & 2.68 & 2.54 & 143 & 228 & 0.0460 \\
4-pole Type B & 11.0 & 10.4 & 3.20 & 3.89 & 1.53 & 2.24 & 2.00 & 174 & 257 & 0.0460
\end{tabular}


lent stiffness of the tilt motion can be expressed as

$$
\begin{aligned}
& \text { 2-pole : } K_{\theta x}=K_{t} k_{p}-K_{r} z_{g} k_{p}-K_{t n}+K_{r p} z_{g}^{2} \\
& \text { 4-pole : } K_{\theta x}=K_{t} k_{p}+K_{r} z_{g} k_{p}-K_{t n}+K_{r p} z_{g}^{2}
\end{aligned}
$$

Because $K_{r p}$ can be regarded as the same level with the equivalent radial stiffness $K_{x}, K_{r p} z_{g}^{2}$ becomes small compared with $K_{\theta x}$, and can be neglected. Substituting the measured results of $K_{\theta x}$ and the corresponding COG position of the type A and B in Eqs. (46) and (47), and subtracting them, we have

$$
\begin{array}{ll}
\text { 2-pole }: & K_{\theta x, A}-K_{\theta x, B}=-K_{r}\left(z_{g, A}-z_{g, B}\right) k_{p} \\
\text { 4-pole }: & K_{\theta x, A}-K_{\theta x, B}=K_{r}\left(z_{g, A}-z_{g, B}\right) k_{p}
\end{array}
$$

From these equations, the radial force gains of $x$-axis can be estimated as

$$
\begin{aligned}
& \text { 2-pole : } K_{r}=1.1[\mathrm{~N} / \mathrm{A}] \\
& \text { 4-pole }: K_{r}=0.34[\mathrm{~N} / \mathrm{A}]
\end{aligned}
$$

where the equivalent stiffness of the 2-pole type A rotor is estimated from Fig. 11 as natural frequency $5.3 \mathrm{~Hz}$ and equivalent stiffness $0.33 \mathrm{Nm} / \mathrm{rad}$. This result shows that the 2-pole rotor has higher radial force gain than that of the 4-pole rotor, and implies the stability of the 2-pole rotor is more sensitive to the COG position.

\subsection{Rotation Tests}

Next, the results of rotation tests are shown. The gains are shown in Table 4. Larger $k_{d}$ was used for rotation tests. Stable rotation was achieved by the 2-pole type B and 4-pole type A rotors using both open and closed torque controllers. However, stable rotation was failed by 2-pole type A and 4-pole type B rotors due to less stability. The 2-pole type B rotor could rotate up to $600 \mathrm{rpm}$, which is under the critical speed in tilt motion. The 4-pole type A rotor could rotate up to 1,500 rpm, which is above the critical speed in radial and tilt motions. The waveforms of the motions and speed of the rotor are shown in Figs. 15, 16, 17, and 18. When the open-loop torque controller was used, the rotating speed was fluctuated, and tilt motions were affected by the difference between the actual angular position and the estimated position. When the closed-loop torque controller was utilized, the tilt motions were better because the actual angular position was used for the current calculation.

\section{Conclusions}

In this paper, a tilt-controlling axial gap self-bearing motor with a single stator was introduced. The theoretical analysis showed that the proposed device generates motor torque, axial force, tilt torques, and radial forces by two kinds of rotating magnetic fluxes. The analysis also showed that motor torque, axial force, and tilt torques could be controlled by the amplitude of the rotating magnetic fluxes. The stability of the radial and tilt motions was discussed, and it was shown that the stability is affected by the COG position of a rotor. The experimental results of levitation and rotation tests verified the analytical results, and both the 2-pole and 4-pole rotors achieved stable levitation and rotation. 



Fig. 15 Results of rotation test with the 2-pole type B rotor and open-loop torque controller. A fluctuation in rotation speed appeared, and the tilt motions were affected.
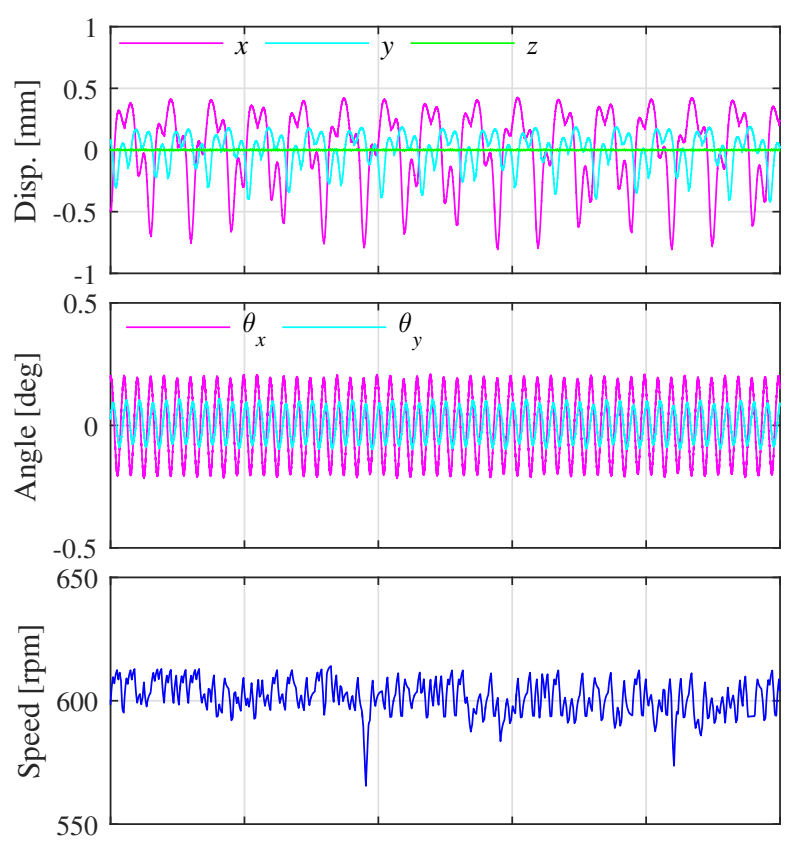

Time [1s/div]

Fig. 16 Results of rotation test with the 2-pole type B rotor and closed-loop torque controller. The fluctuation disappeared, and the tilt motions were improved.

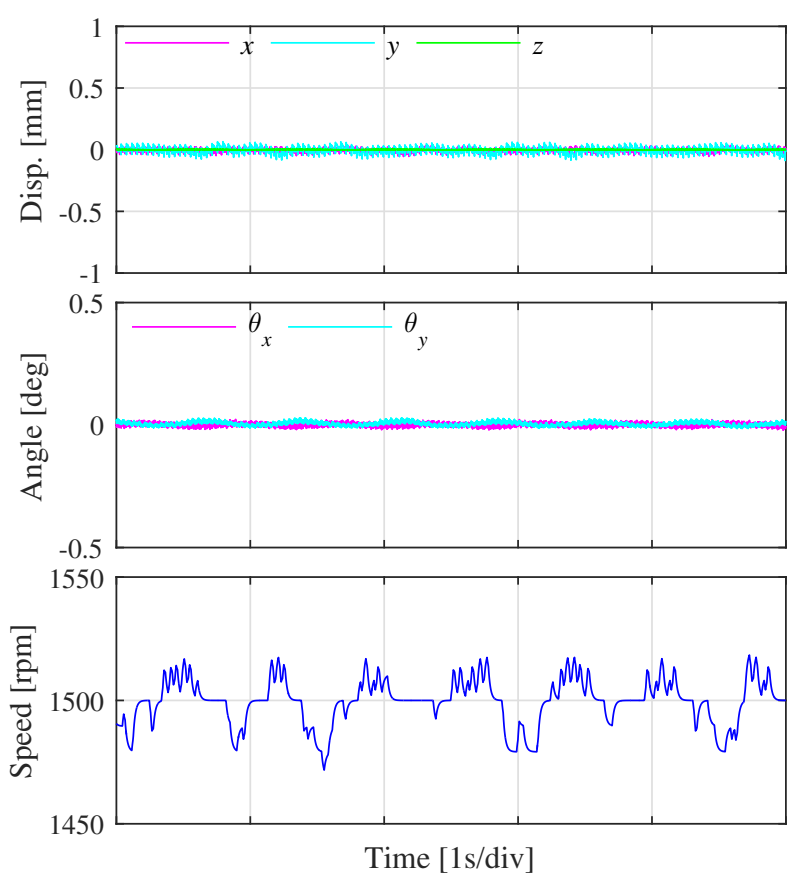

Fig. 17 Results of rotation test with the 4-pole type A rotor and open-loop torque controller. The rotor could rotate over critical speed.
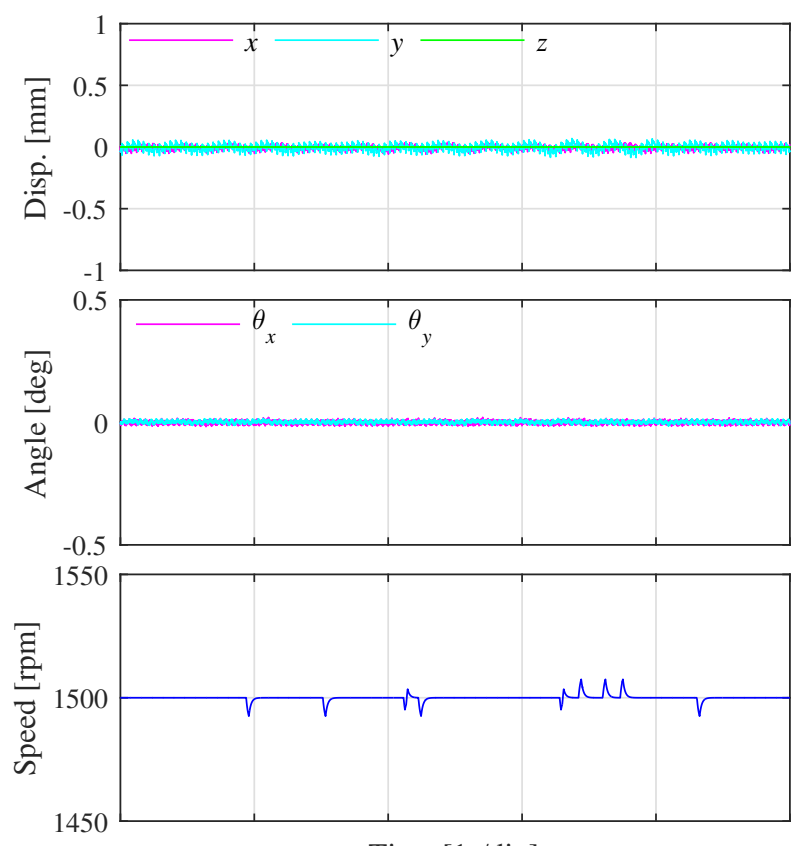

Time $[1 \mathrm{~s} / \mathrm{div}]$

Fig. 18 Results of rotation test with the 4-pole type A and closedloop torque controller. Stable levitation and rotation were achieved.

Table 4 Parameters of PID and PI controllers for rotation tests. Values not mentioned in the table were the same as the levitation tests.

\begin{tabular}{cccc} 
& $k_{p}$ & $k_{d}$ & $k_{i}$ \\
\hline$\theta_{x}$ & & $2[\mathrm{As} / \mathrm{rad}]$ & \\
$\theta_{y}(2$-pole $)$ & & $5[\mathrm{As} / \mathrm{rad}]$ & \\
$\theta_{y}(4-$ pole $)$ & & $2[\mathrm{As} / \mathrm{rad}]$ & \\
$\omega$ & $0.03[\mathrm{~A} / \mathrm{rpm}]$ & - & $0.01[\mathrm{~A} / \mathrm{rpm} \mathrm{s}]$
\end{tabular}




\section{References}

Asama, J. and Chiba, A., Development of One-Axis Actively Regulated Bearingless Motor with a Repulsive Type Passive Magnetic Bearing, Proc. of The 2014 International Power Electronics Conference, Hiroshima, Japan, (2014), pp. 988-993.

Bauer, W. and Amrhein, W., Electrical Design and Winding Selection for a Bearingless Axial Force/Torque Motor, Proc. of International Symposium on Power Electronics Power Electronics, Electrical Drives, Automation and Motion, Sorrento, Italy, (2012), pp. 1224-1229.

Chiba, A., Fukao, T., Ichikawa, O., Oshima, M., Takemoto, M. and Dorrell, D.G., Magnetic Bearings and Bearingless Drives, ELSEVIER (2005).

Kurita, N., Ishikawa, T., Saito, N. and Masuzawa, T., Basic Design of the Maglev Pump for Total Artificial Heart by using Double Stator Type Axial Self-bearing Motor, Proc. of The 15th International Symposium on Magnetic Bearings, Kitakyushu, Japan, (2016), pp. 509-514.

Mitterhofer, H. and Jungmayr, G., Stability improvement of passively stabilized degrees of freedom in magnetically levitated systems, Proc. of The 15th International Symposium on Magnetic Bearings, Kitakyushu, Japan, (2016), pp. 701-708.

Matsuoka, A., Sumino, M., Ueno, S. and Takeda, T., Development of High-speed Motor at Extremely Low Temperatures with Axial Self-bearing Motor and Superconducting Magnetic Bearing, International Journal of Applied Electromagnetics and Mechanics, Vol.45(2014), pp.859-865.

Nguyen, Q. D. and Ueno, S., Analysis and Control of Non-Salient Permanent Magnet Axial-Gap Self-Bearing Motor, IEEE Transactions on Industrial Electronics, Vol.58, No.7(2011), pp. 2644-2652.

Okamura, T., Ueno, S., Sakagami, M. and Tanaka, S., Efficiency of Small Regenerative Pump Using Axial Self-bearing Motor, Vol. 76, No. 772 (2010), pp. 3535-3541.(in Japanese)

Osa, M., Masuzawa, T., Omori, N. and Tatsumi, E., 5-DOF Control Miniaturized Self-bearing Motor for Pediatric Ventricular Assist Device, The 22nd MAGDA Conference in Miyazaki, (2013), pp. 280-298. (in Japanese)

Sugimoto, H., Shimura, I. and Chiba, A., Design Consideration for Performance Improvement in One-Axis Actively Positioned Single-Drive Bearingless Motor, Proc. of The 15th International Symposium on Magnetic Bearings, Kitakyushu, Japan, (2016), pp. 441-445.

Sumino, M. and Ueno, S., Tilt control with axial self-bearing motor, Transactions of the JSME, Vol.80, No.815 (2014a), DOI: 10.1299/transjsme.2014dr0207. (in Japanese)

Sumino, M. and Ueno, S., Rotation Test of a Tilt-Controlling Axial Self-Bearing Motor with Superconducting Magnetic Bearing, Proc. of The 12th International Conference on Motion and Vibration Control, Sapporo, Japan, 1D13 (2014b).

Tomita, I., Ichikaw, O., Michioka, C., Chiba, A. and Fukao, T., Decoupling Controller for Inclination Control of Shaftless Axial-gap Bearingless Motors, Proc. of the 9th SEAD, Sapporo, Japan, (1997), pp. 529-534. (in Japanese)

Ueno, S. and Okada, Y., Characteristics of Axial Force and Rotating Torque and Their Control ofPermanent Magnet Type Axial Gap Self-Bearing Motor, Electrical Engineering in Japan, Vol. 132, No. 1 (2000), pp. 81-91. 